\title{
Highly Sensitive and Selective Sodium Ion Sensor Based on Silicon Nanowire Dual Gate Field-Effect Transistor
}

\author{
Seong-Kun Cho (D) and Won-Ju Cho *(D)
}

check for updates

Citation: Cho, S.-K.; Cho, W.-J. Highly Sensitive and Selective Sodium Ion Sensor Based on Silicon Nanowire Dual Gate Field-Effect Transistor. Sensors 2021, 21, 4213. https://doi.org/10.3390/s21124213

Academic Editors: Marco Mugnaini and Ada Fort

Received: 14 May 2021

Accepted: 17 June 2021

Published: 19 June 2021

Publisher's Note: MDPI stays neutral with regard to jurisdictional claims in published maps and institutional affiliations.

Copyright: (c) 2021 by the authors. Licensee MDPI, Basel, Switzerland. This article is an open access article distributed under the terms and conditions of the Creative Commons Attribution (CC BY) license (https:// creativecommons.org/licenses/by/ $4.0 /)$.
Department of Electronic Materials Engineering, Kwangwoon University, 20, Gwangun-ro, Nowon-gu, Seoul 01897, Korea; whtjdrms98@gmail.com

* Correspondence: chowj@kw.ac.kr; Tel.: +82-940-5163

\begin{abstract}
In this study, a highly sensitive and selective sodium ion sensor consisting of a dualgate (DG) structured silicon nanowire (SiNW) field-effect transistor (FET) as the transducer and a sodium-selective membrane extended gate (EG) as the sensing unit was developed. The SiNW channel DG FET was fabricated through the dry etching of the silicon-on-insulator substrate by using electrospun polyvinylpyrrolidone nanofibers as a template for the SiNW pattern transfer. The selectivity and sensitivity of sodium to other ions were verified by constructing a sodium ion sensor, wherein the EG was electrically connected to the SiNW channel DG FET with a sodium-selective membrane. An extremely high sensitivity of $1464.66 \mathrm{mV} / \mathrm{dec}$ was obtained for a $\mathrm{NaCl}$ solution. The low sensitivities of the SiNW channel FET-based sodium ion sensor to $\mathrm{CaCl}_{2}, \mathrm{KCl}$, and $\mathrm{pH}$ buffer solutions demonstrated its excellent selectivity. The reliability and stability of the sodium ion sensor were verified under non-ideal behaviors by analyzing the hysteresis and drift. Therefore, the SiNW channel DG FET-based sodium ion sensor, which comprises a sodium-selective membrane EG, can be applied to accurately detect sodium ions in the analyses of sweat or blood.
\end{abstract}

Keywords: sodium ion sensor; silicon nanowire; dual-gate field-effect transistor; sodium-selective membrane; extended gate

\section{Introduction}

Human sweat contains key biomarkers such as glucose, lactate, $\mathrm{Na}^{+}, \mathrm{K}^{+}, \mathrm{H}^{+}, \mathrm{NH}^{4+}$, $\mathrm{Ca}^{2+}$, and $\mathrm{Cl}^{-}$that correlate with their respective concentrations in blood. Hence, human sweat can be analyzed to conduct real-time, non-invasive personalized health monitoring [1,2]. In particular, it is important to monitor these analytes in patients suffering from complex body conditions, which range from hydration to heart and metabolic diseases. As active electronic devices, field-effect transistor (FET)-based ion sensors are promising candidates for such non-invasive applications owing to their numerous advantages, such as small sizes, portability, and rapid detection [3-6]. A high-performance ion sensor must possess high sensitivity, stability, and reliability in order to ensure its successful implementation. In addition, the presence of several ions in analytes such as human sweat or blood necessitates high selectivity to ensure that only the target ions are detected. Conventional bulk silicon FET-based sensors with a single-gate (SG) structure exhibit a Nernstian limit of $59.16 \mathrm{mV} / \mathrm{dec}$, which is the theoretical upper limit of sensitivity at room temperature [7,8]. FET-based sensors with dual-gate (DG) structures on silicon-on-insulator (SOI) substrates have also attracted significant attention. In DG-structured ion-sensitive (IS) FETs, capacitive coupling occurs between the top and bottom gate oxide layers of the channel due to structural specificity; this amplifies the sensitivity of these devices [9-14]. The amplification ratio increases through capacitive coupling in the DG sensing mode with the increased capacitance of the top gate oxide. We recently reported that a DG-structured silicon nanowire (SiNW) channel FET-based $\mathrm{pH}$ sensor exhibits better sensitivity, detection capability, stability, and reliability than conventional FET-based sensors [15]. In previous 
studies, high sensitivity to $\mathrm{pH}$ values was achieved using an $\mathrm{SnO}_{2}$ sensing membrane based on the site-binding theory.

In this paper, we propose a highly sensitive sodium ion-selective sensor based on a SiNW channel DG FET. The sensing unit of the extended gate (EG) with a sodiumselective membrane and the transducer unit of the SiNW channel DG FET were built independently. These two units were electrically connected in series to complete the sodium ion sensor platform. The SiNW channel DG FET transducer unit was fabricated through the dry etching of SOI substrates by using electrospun polyvinyl pyrrolidone (PVP) nanofibers (NFs) as a template for the SiNW pattern transfer. The fundamental electrical characteristics and signal amplification functions of the fabricated SiNW channel DG FET were subsequently evaluated. In addition, the sodium-selective membrane EG detects only sodium ions with an excellent selectivity; this is because the sodium ionophore of the membrane can selectively capture sodium ions alone. The sensitivity and ion selectivity of the sodium ion sensor were verified by using $\mathrm{NaCl}, \mathrm{CaCl}_{2}, \mathrm{KCl}$, and $\mathrm{pH}$ buffer solutions. Furthermore, the reliability and stability of the sodium ion sensor platform were evaluated by analyzing non-ideal behaviors, such as the hysteresis and drift.

\section{Materials and Methods}

\subsection{Fabrication of SiNWs on SOI Wafer}

The SiNWs were fabricated on a fully depleted p-type (100) SOI wafer with a doping concentration of $1 \times 10^{15} \mathrm{~cm}^{-3}$, a 750-nm-thick buried oxide (BOX) layer, and a 70-nm-thick top Si layer. The SiNWs were fabricated by transferring the pattern template of the PVP NFs to the uppermost Si layer of the SOI wafer. The PVP NF precursor solution used for electrospinning was prepared by stirring $100 \mathrm{mg}$ of PVP $\left(\mathrm{M}_{\mathrm{W}} \approx 1,300,000\right.$, Sigma-Aldrich Co., St. Louis, MO, USA) in $1.5 \mathrm{~mL}$ of ethanol ( $\geq 99.7 \%)$ at $800 \mathrm{rpm}$ for $2 \mathrm{~h}$ at room temperature. A schematic of the electrospinning system is shown in Figure 1a. It consists of a syringe with a spinning needle, a syringe pump (NE-1000; New Era Pump Systems Inc., Farmingdale, NY, USA), a high-voltage power supply, and a grounded collector $\left(15 \times 15 \mathrm{~cm}^{2}\right.$ copper plate). The PVP NFs were electrospun onto the SOI substrate by loading a syringe with the PVP NF precursor solution and fixing it horizontally to the syringe pump. The pressure of the syringe pump was $0.4 \mathrm{~mL} / \mathrm{h}$; the inner diameter of the needle was $0.635 \mathrm{~mm}$; the working distance between the needle and the grounded collector was $20 \mathrm{~cm}$; the positive voltage was adjusted to $20 \mathrm{kV}$; and the relative temperature and humidity were maintained at $25{ }^{\circ} \mathrm{C}$ and $25 \%$, respectively. Calcination was performed by using a resistive heating furnace at $300^{\circ} \mathrm{C}$ for $1 \mathrm{~h}$ in air to remove the solvent in the electrospun PVP NFs. The equilibrium-melting temperature of PVP is $300^{\circ} \mathrm{C}$; thus, the abovementioned process improves the thermomechanical stability of the template fibers and the adhesion between the PVP NFs and the substrate surface [16]. An optical microscopy image of the electrospun PVP NFs, which were used as hard masks for the silicon dry etching, is presented in the inset of Figure 1a. A section of the uppermost Si layer was selectively etched by exposing the electrospun SOI substrate with the PVP NFs to the $\mathrm{SF}_{6}$ plasma of a reactive ion etching (RIE) system. The power of the plasma treatment in the RIE process was $50 \mathrm{~W}$; the $\mathrm{SF}_{6}$ gas flow and pressure were fixed at $20 \mathrm{sccm}$ and $300 \mathrm{mTorr}$, respectively; and the process duration was $15 \mathrm{~s}$. The residual PVP NFs were removed by using a sulfuric acid-hydrogen peroxide mixture solution after the $\mathrm{SF}_{6}$ plasma etching process. A schematic of the fabrication of the SiNWs on an SOI substrate is shown in Figure $1 \mathrm{~b}$. Scanning electron microscopy (SEM) images of the patterned SiNWs are presented in Figure 2. 


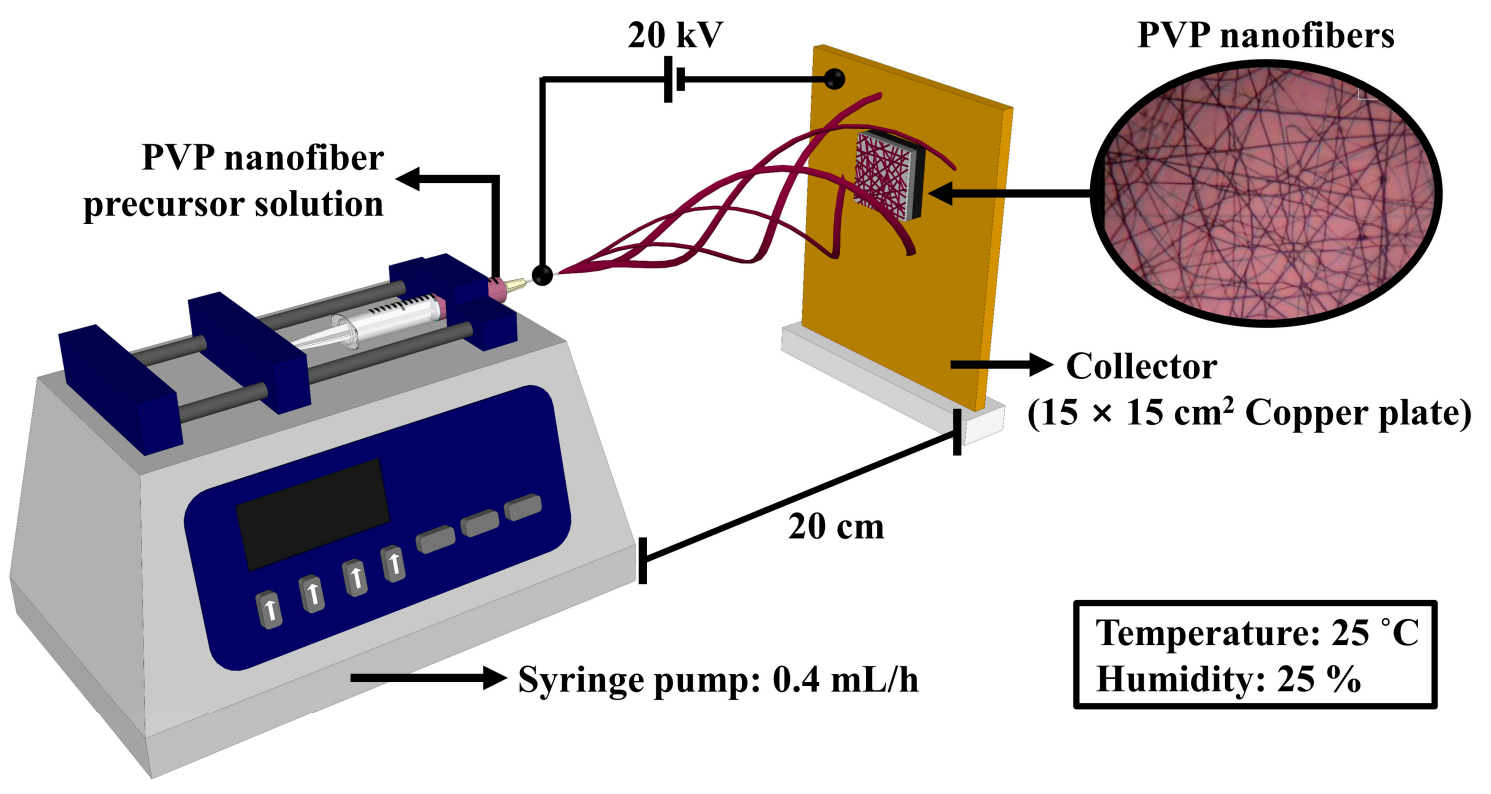

(a)
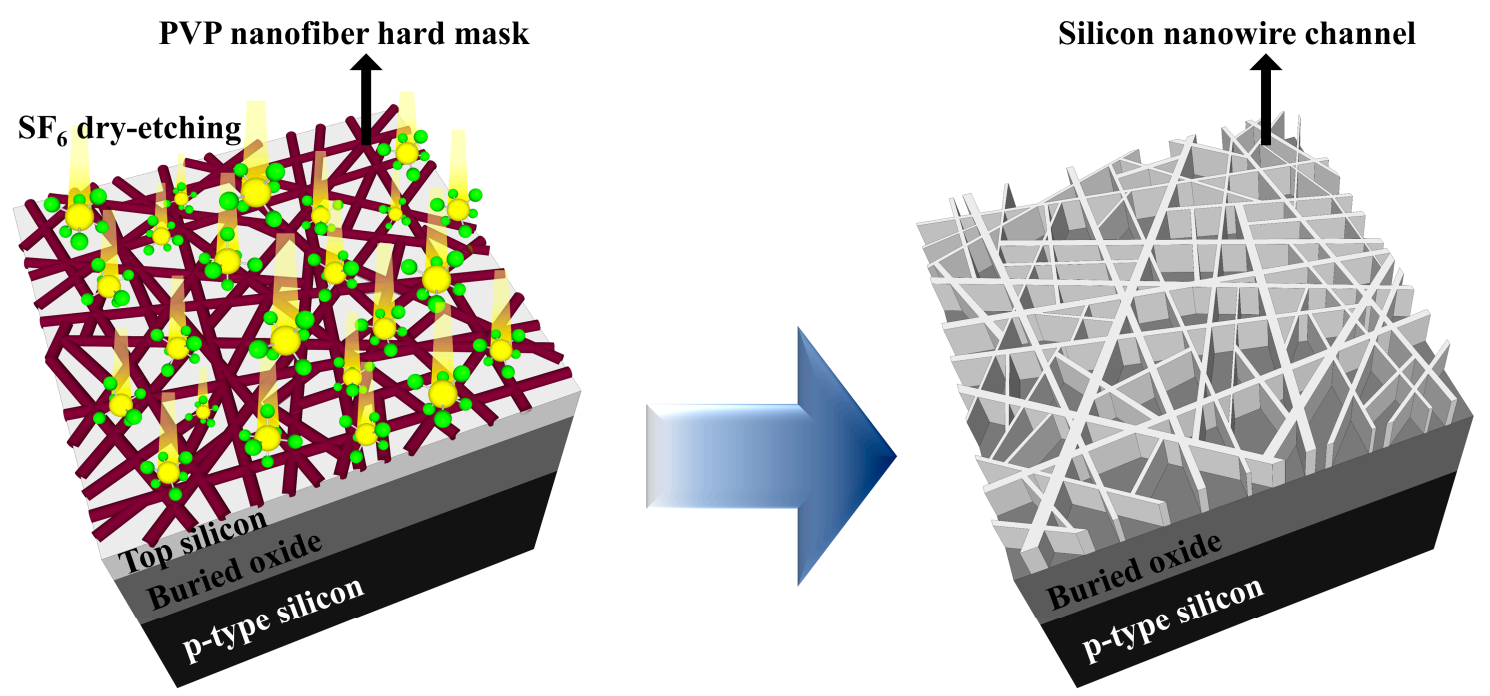

(b)

Figure 1. (a) Schematic of the electrospinning system. (b) Fabrication of SiNWs on an SOI substrate.

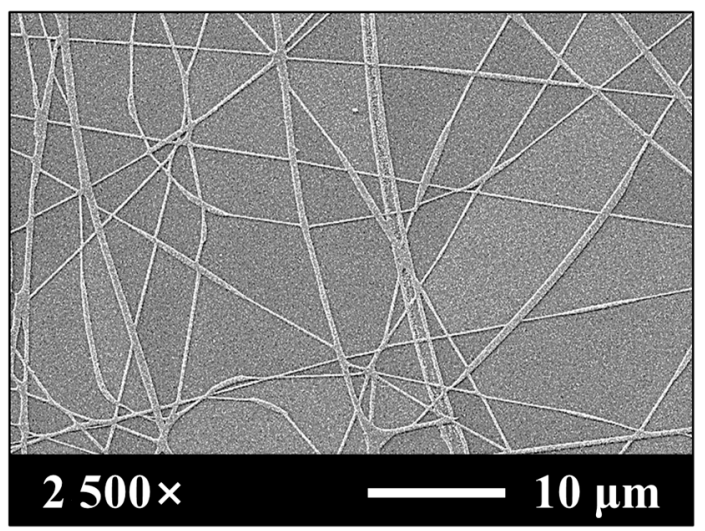

(a)

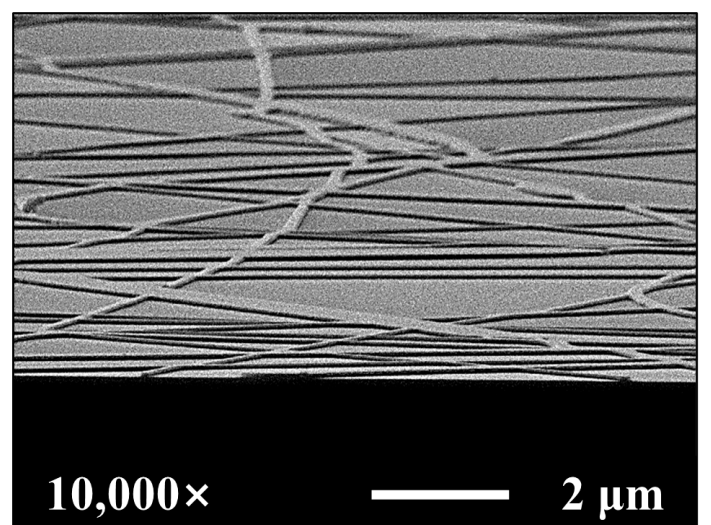

(b)

Figure 2. (a) Top view and (b) tilted view SEM images of the SiNWs after transferring the pattern template of the PVP NFs to the uppermost Si layer of the SOI wafer. 


\subsection{Fabrication of SiNW Channel DG Field-Effect Transistors}

The SiNW channel DG FET transducer unit was fabricated by processing the SOI wafer into the SiNW through the transfer of the pattern template of the PVP NFs. The active regions of the FET, which had a channel width and length of 20 and $10 \mu \mathrm{m}$, respectively, were formed through photolithography and RIE etching after standard RCA cleaning. Highly doped source/drain regions were selectively formed through the solid-phase diffusion of phosphorus from phosphosilicate glass films with rapid thermal annealing at $950{ }^{\circ} \mathrm{C}$. A 20-nm-thick $\mathrm{SiO}_{2}$ layer was deposited as the top gate oxide layer through $\mathrm{RF}$ magnetron sputtering. This was followed by the formation of the top gate and the source/drain electrodes, which were 150-nm-thick Al films, through a lift-off process employing an electron beam evaporator. The p-type substrate under the BOX layer of the SOI serves as the bottom gate. Forming gas annealing treatment was performed at $450{ }^{\circ} \mathrm{C}$ for 30 min under a $2 \% \mathrm{H}_{2} / \mathrm{N}_{2}$ ambient in order to improve the electrical properties and stability of the fabricated SiNW channel DG FET transducer. The schematic of the SiNW channel DG FET transducer unit is presented in Figure 3a.

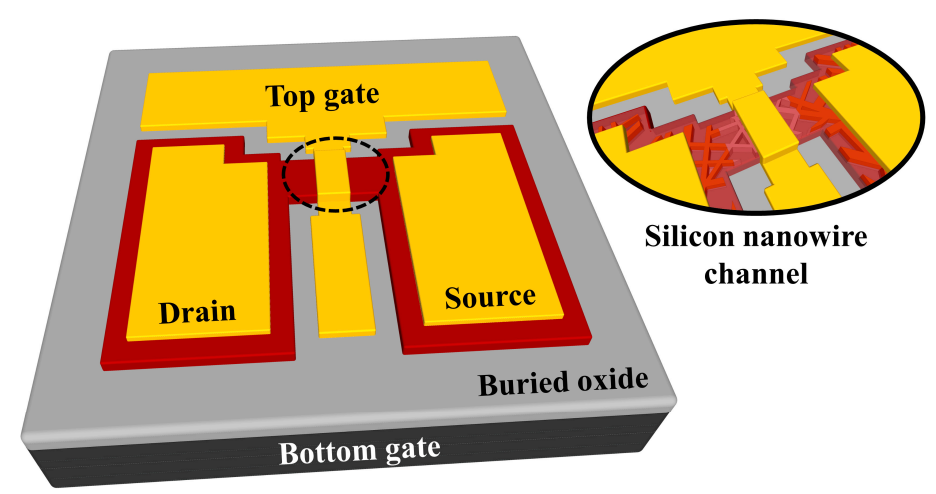

(a)

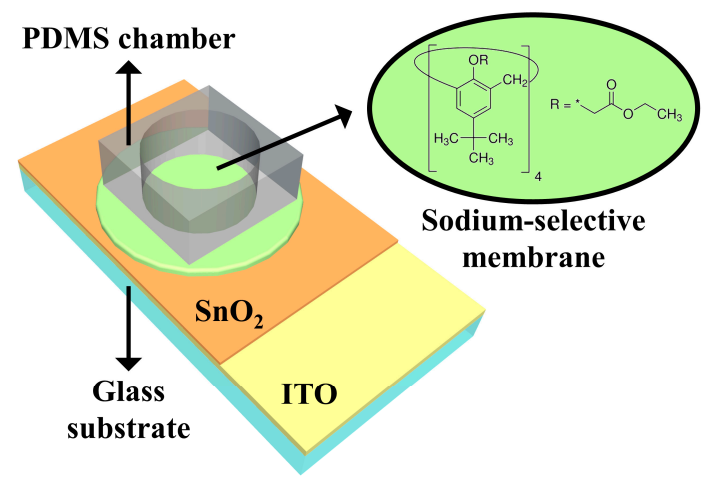

(b)

Figure 3. Schematic of the (a) SiNW channel DG FET transducer unit and the (b) sodium-selective membrane EG sensing unit.

\subsection{Fabrication of Sodium-Selective Membrane EG}

The sodium-selective membrane EG sensing unit was built by using a $1.5 \times 2.5 \mathrm{~cm}^{2}$ glass substrate (Corning Inc., New York, NY, USA) to reduce the overall cost of the experiment. A 300-nm-thick ITO conductive film and a 50-nm-thick $\mathrm{SnO}_{2}$ film were sequentially deposited through RF magnetron sputtering after cleaning the glass substrate ultrasonically. The sodium-selective membrane was formed by drop-casting $100 \mu \mathrm{L}$ of the membrane cocktails onto the $\mathrm{SnO}_{2}$ film and drying it for $24 \mathrm{~h}$. The sodium-selective membrane cocktail was prepared by stirring $100 \mathrm{mg}$ of a mixture of $\mathrm{Na}$ ion channel stage $\mathrm{X}(1 \% w / w)$, sodium tetrakis [3,5bis(trifluoromethyl)phenyl] borate (Na-TFPB) $(0.55 \% w / w)$, polyvinyl chloride (PVC) $(33 \% w / w)$, and bis(2-ethylhexyl) sebacate (DOS) $(65.45 \% w / w)$ in $660 \mu \mathrm{L}$ of tetrahydrofuran (THF) at $800 \mathrm{rpm}$ for $4 \mathrm{~h}$ at room temperature. In the stacked structure of the ITO/ $\mathrm{SnO}_{2}$ / sodium-selective membrane, the surface potential change of the $\mathrm{SnO}_{2}$ film due to the ion capture of the sodium-selective membrane is transferred directly to the uppermost Al gate electrode of the FET through the ITO conductive film. An EG sensing unit was developed by attaching a polydimethylsiloxane (PDMS) chamber with an inner diameter of $0.6 \mathrm{~cm}$ to the top of the sodium-selective membrane for solution storage. The reagents were purchased from Sigma-Aldrich Co. (St. Louis, MO, USA) and used without additional purification. A schematic of the sodium-selective membrane EG sensing unit is shown in Figure 3b, and the molecular structure of the Na ionophore $X$ in the sodium-selective membrane is depicted in the inset of Figure 3b. 


\subsection{Characterization of SiNW Channel DG FET and Sodium Ion-Selective Sensor}

The current-voltage (I-V) characteristics of the fabricated SiNW channel DG FETs and the sensing properties of the sodium ion sensor, which was constructed by using the SiNW channel DG FET and the sodium-selective membrane EG, were measured using an Agilent 4156B precision semiconductor parameter analyzer. The electrical measurements of these devices were carried out in an electromagnetically shielded dark box to reduce external disturbances, such as noise signals and light. A commercial $\mathrm{Ag} / \mathrm{AgCl}$ reference electrode (2086A-06T, Horiba, Kyoto, Japan), which was composed of a ceramic-plug junction and an internal solution saturated with $\mathrm{KCl}$ and $\mathrm{AgCl}$, was used as the reference electrode to detect ions in the solution. The sensing electrolyte was obtained by dissolving $\mathrm{NaCl}$, $\mathrm{KCl}$, and $\mathrm{CaCl}_{2}$ (Sigma-Aldrich Co., St. Louis, MO, USA) in DI water to prepare varying concentrations of $\mathrm{NaCl}, \mathrm{KCl}$, and $\mathrm{CaCl}_{2}$ solutions, in addition to a commercial $\mathrm{pH}$ buffer solution (SAMCHUN CHEMICAL, Pyeongtaek, Korea) containing potassium hydrogen phthalate. Figure 4 shows the electrical connection between the SiNW channel DG FET and the sodium-selective membrane EG for the sodium ion-selective sensor platform. The FET transducer unit has independent gates at the top and bottom of the SiNW channel, thereby enabling the sensing operation through the top or bottom gates. The operation of the top gate, as shown in Figure 4a, was termed as the SG sensing mode, whereas the operation of the bottom gate, as shown in Figure $4 \mathrm{~b}$, was termed as the DG sensing mode. The SG sensing mode involved a voltage sweep of the reference electrode connected to the top gate, while the bottom gate was grounded. The DG sensing mode involved a voltage sweep of the bottom gate, while the reference electrode connected to the top gate was grounded. The sensitivity of the sodium ion sensor was determined by varying the voltage in the top or bottom gates (reference voltage, $\mathrm{V}_{\text {ref }}$ ) at a drain current of $1 \mathrm{nA}$ (reference current, $\mathrm{I}_{\text {ref }}$ ) depending on the concentration of the $\mathrm{NaCl}$ solution. In addition, the hysteresis and drift effects were measured to evaluate the non-ideal behaviors of the sodium ion sensor. The hysteresis voltage was equal to the difference between the $V_{\text {ref }}$ of the first and last $10^{-4} \mathrm{M}$ in the concentration loop of $10^{-4} \rightarrow 10^{-3} \rightarrow 10^{-2} \rightarrow 10^{-1} \rightarrow 10^{0} \rightarrow 10^{-1} \rightarrow 10^{-2} \rightarrow 10^{-3}$ $\rightarrow 10^{-4} \mathrm{M}$. The drift rate was equal to the magnitude of change in $\mathrm{V}_{\text {ref }}\left(\Delta \mathrm{V}_{\text {ref }}\right)$ due to the exposure of the sodium-selective membrane to a $10^{-4} \mathrm{M} \mathrm{NaCl}$ solution for $10 \mathrm{~h}$.

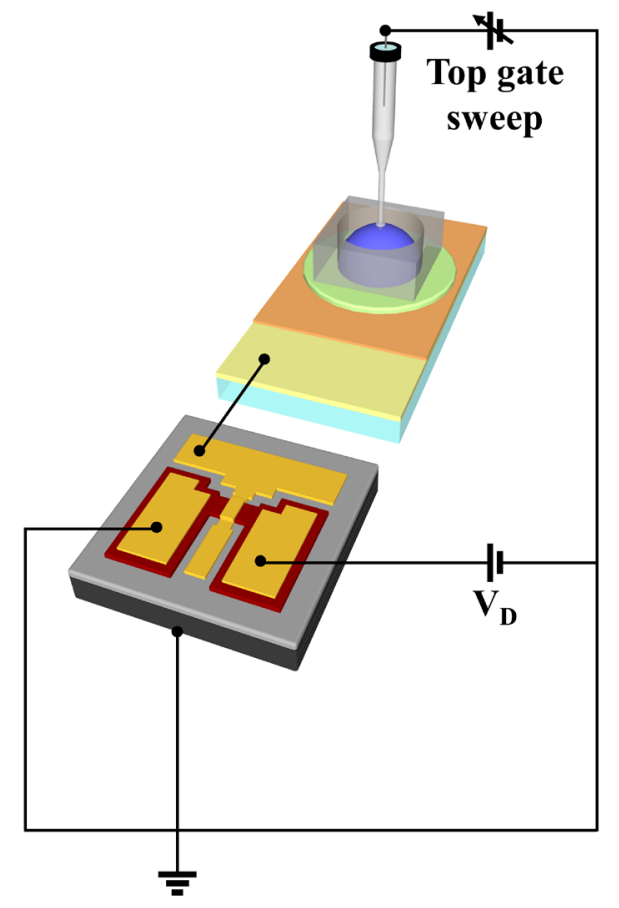

(a)

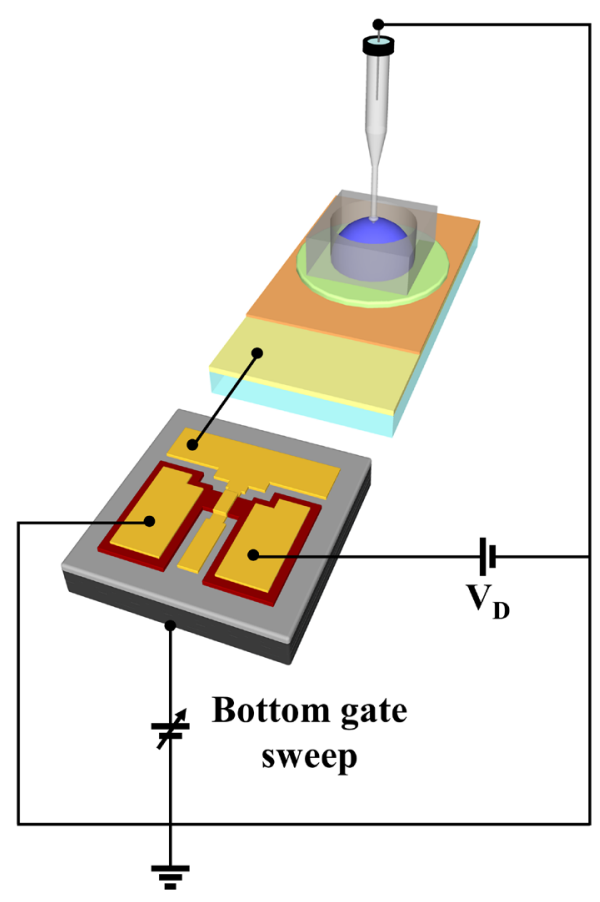

(b)

Figure 4. Schematic of the (a) SG mode and (b) DG mode sensing operations. 


\section{Results and Discussion}

\subsection{Electrical Characteristics of SiNW Channel DG FETs}

Figure 5 shows the transfer characteristic $\left(\mathrm{I}_{\mathrm{D}}-\mathrm{V}_{\mathrm{G}}\right)$ curves and output characteristic $\left(\mathrm{I}_{\mathrm{D}}-\mathrm{V}_{\mathrm{D}}\right)$ curves (insets) of the SiNW channel DG FETs, where Figure $5 \mathrm{a}, \mathrm{b}$ correspond to the top gate and bottom gate operation modes, respectively. The transfer characteristics were measured at drain voltages $\left(\mathrm{V}_{\mathrm{D}}\right)$ of $50 \mathrm{mV}$ and $1 \mathrm{~V}$ for the top gate and bottom gate operation modes, respectively. The top gate voltage was varied from -1.0 to $1.5 \mathrm{~V}$ (in steps of $25 \mathrm{mV}$ ) to measure the transfer characteristics of the top gate operation. Similarly, the bottom gate voltage was varied from -15 to $35 \mathrm{~V}$ (in steps of $500 \mathrm{mV}$ ) for the bottom gate operation. The output characteristics were obtained by varying the top gate voltage from 0 to $1 \mathrm{~V}$ (in steps of $0.1 \mathrm{~V}$ ) and the bottom gate voltage from 0 to $30 \mathrm{~V}$ (in steps of $3 \mathrm{~V}$ ). As a result, the SiNW channel FETs, which were based on the electrospun PVP NF pattern template transfer method, exhibited excellent electrical characteristics during the top and bottom gate operations.

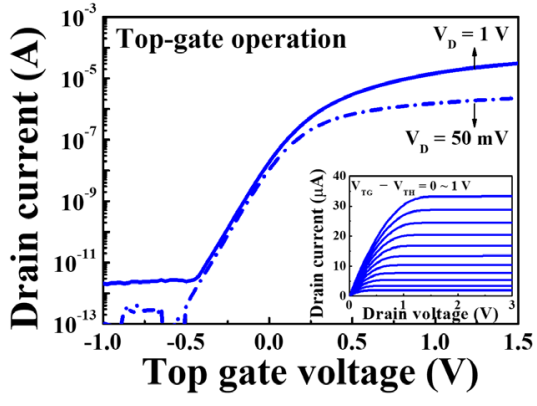

(a)

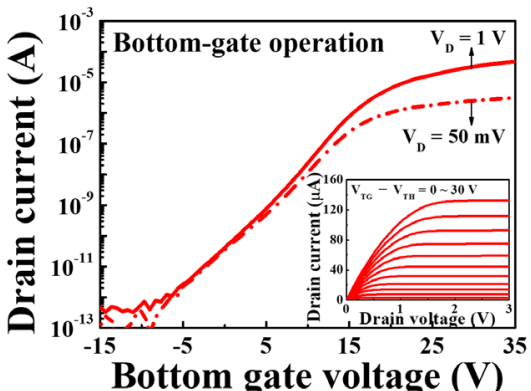

(b)

Figure 5. Transfer characteristic curves for the SiNW channel DG FETs operated by the (a) top gate and (b) bottom gate. The insets display the output characteristic curves.

Table 1 lists the electrical parameters extracted from the transfer characteristic curves of the SiNW channel DG FETs. A threshold voltage $\left(\mathrm{V}_{\mathrm{TH}}\right)$ of $-0.3 \mathrm{~V}$, field-effect mobility $\left(\mu_{\mathrm{FE}}\right)$ of $713.1 \mathrm{~cm}^{2} / \mathrm{V} \cdot \mathrm{s}$, sub-threshold swing (SS) of $96.9 \mathrm{mV} / \mathrm{dec}$, and an on/off current ratio $\left(\mathrm{I}_{\mathrm{ON}} / \mathrm{I}_{\mathrm{OFF}}\right)$ of $3.0 \times 10^{7}$ were obtained during the top gate operation. Similarly, the values of $\mathrm{V}_{\mathrm{TH}}, \mu_{\mathrm{FE}}, \mathrm{SS}$, and $\mathrm{I}_{\mathrm{ON}} / \mathrm{I}_{\mathrm{OFF}}$ were $-2.0 \mathrm{~V}, 956.1 \mathrm{~cm}^{2} / \mathrm{V} \cdot \mathrm{s}, 713.8 \mathrm{mV} / \mathrm{dec}$, and $6.4 \times 10^{7}$, respectively, under the bottom gate operation. These values confirm the excellent electrical performance of the SiNW channel DG FET, which is the basic component of high-sensitivity SG or DG mode sensing and selective sodium sensors.

Table 1. Electrical parameters of the SiNW channel DG FETs during top and bottom gate operations.

\begin{tabular}{ccccc}
\hline Operation Mode & $\mathbf{V}_{\mathbf{T H}}(\mathbf{V})$ & $\boldsymbol{\mu}_{\mathrm{FE}}\left(\mathbf{c m}^{2} / \mathbf{V} \cdot \mathbf{s}\right)$ & $\mathbf{S S}(\mathbf{m V} / \mathbf{d e c})$ & $\mathbf{I}_{\mathbf{O N}} / \mathbf{I}_{\mathbf{O F F}}(\mathbf{A} / \mathbf{A})$ \\
\hline Top gate & -0.3 & 713.1 & 96.9 & $3.0 \times 10^{7}$ \\
Bottom gate & -2.0 & 956.1 & 713.8 & $6.4 \times 10^{7}$ \\
\hline
\end{tabular}

Additionally, to investigate the effect on noise, we tested the stability of the two modes, as shown in Figure 6, by applying a $0.4 \mathrm{~V}$ amplitude square wave pulse (assuming noise) to the gate voltage sweep. As a result, $\mathrm{I}_{\max } / \mathrm{I}_{\min }=78.36$ in the SG mode, while $\mathrm{I}_{\max } / \mathrm{I}_{\min }=1.3$ in the DG mode. This is because the S.S of the transfer characteristic curve in SiNW channel DG mode is $713.8 \mathrm{mV} / \mathrm{dec}$, which is larger than the $\mathrm{SS}$ of $96.9 \mathrm{mV} / \mathrm{dec}$ in SG mode, and the fluctuation of the drain current with respect to the pulse (noise) of the gate voltage is relatively small. Therefore, we verified that SiNW channel DG FET have better noise tolerance than conventional SG FETs. 


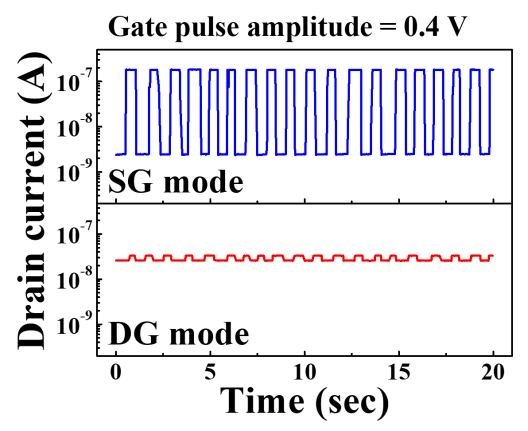

Figure 6. The fluctuation of drain current due to square wave pulse (assuming noise) applied to the voltage sweep under SG and DG operation mode.

\subsection{Signal Amplification Capabilities of SiNW Channel DG FETs}

The maximum sensitivity of an SG FET-based sensor is limited to $59.16 \mathrm{mV} / \mathrm{dec}$ at room temperature, because the sensitivity $\left(\Delta V_{T H}^{t o p}\right)$ is determined according to the surface potential $\left(\Delta \psi_{0}\right)$ of the sensing membrane: $\Delta V_{T H}^{\text {top }}=-\Delta \psi_{0}[17,18]$. This value is known as the Nernstian limit. The sensitivity of a DG FET-based sensor can also be amplified through capacitive coupling between the top and bottom gate oxides of the channel due to their structural specificity [17-19].

$$
\Delta V_{T H}^{\text {bottom }}=-\frac{C_{\text {top }}}{C_{\text {bottom }}} \Delta \psi_{0}=\frac{C_{\text {top }}}{C_{\text {bottom }}} \Delta V_{T H^{\prime}}^{\text {top }}
$$

where $C_{\text {top }}$ and $C_{\text {bottom }}$ represent the capacitance per unit area of the top and bottom gates, respectively. $\Delta V_{T H}^{\text {bottom }}$ and $\Delta V_{T H}^{\text {top }}$ represent the change in the $V_{T H}$ of the bottom and top gates, respectively; these are indicators of sensitivity. The abovementioned relationship suggests that sensitivity can significantly exceed the Nernstian limit if an appropriate value of $\Delta V_{T H}^{\text {bottom }}$ and a suitable combination of $C_{\text {top }}$ and $C_{\text {bottom }}$ are employed. The capacitive coupling ratio, which is essentially the ratio of the amplification, increases if the magnitude of $C_{\text {top }}$ is greater than that of $C_{\text {bottom }}$. As can be seen from Equation (1), reducing the thickness of the top gate oxide increases $C_{\text {top }}$, which improves the capacitive coupling. However, as the thickness of the top gate oxide decreases, reliability issues arise owing to the increased leakage current or the poor dielectric breakdown properties of the thin gate insulator. Meanwhile, the SiNW-type channel has a larger specific surface area than the conventional planar film-type channel, resulting in a larger $C_{\text {top }}$. Therefore, the SiNWtype channel is an effective structure that can improve the capacitive coupling without reducing the thickness of the top gate oxide. Thus, SiNW-type channels feature larger capacitive coupling ratios than film-type channels; this has also been confirmed in a previous study [15]. The transfer characteristics during the bottom gate operation were measured by using a constant top gate bias to evaluate the signal amplification capabilities of the SiNW channel DG FETs, as shown in Figure 7a. The top gate bias was varied from +600 to $-600 \mathrm{mV}$ in steps of $300 \mathrm{mV}$. A reduction in the top gate bias gradually shifts the transfer characteristic curve to a relatively large bottom gate voltage. Figure $7 \mathrm{~b}$ shows the variation in the top gate bias with the $\Delta \mathrm{V}_{\text {ref }}$ of the SiNW channel DG FETs, which was obtained from the transfer characteristic curves. The ratio of the change in $\Delta \mathrm{V}_{\text {ref }}$ to the top gate bias, which represents the capacitive coupling ratio of the SiNW channel DG FETs, was 26.3. This implies that the SiNW channel DG FET can amplify the conventional sensitivity by a factor of 26.3 , thereby improving the sensitivity in the DG sensing mode significantly. 


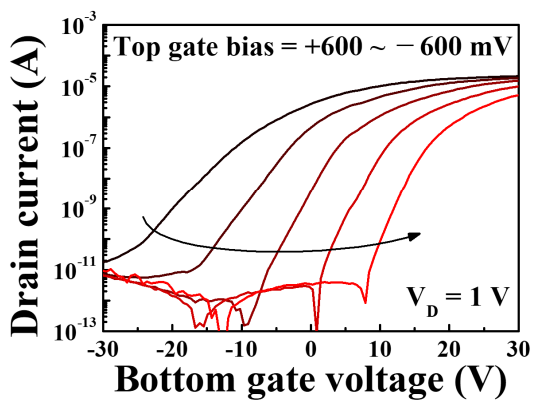

(a)

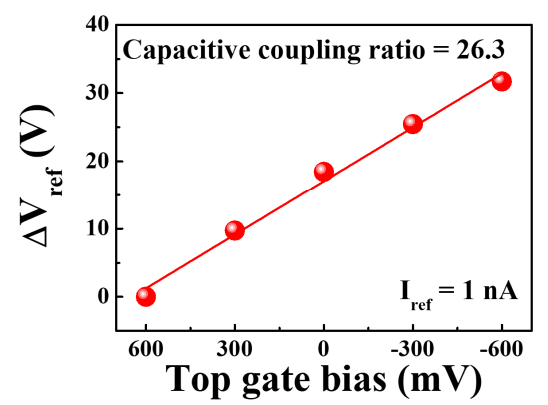

(b)

Figure 7. (a) Transfer characteristic curves of the SiNW channel DG FET during the bottom gate operation with a constant top gate bias varying from +600 to $-600 \mathrm{mV}$ (in steps of $300 \mathrm{mV}$ ). (b) Variation in the top gate bias with $\Delta \mathrm{V}_{\text {ref. }}$.

\subsection{Signal Amplification Capabilities of SiNW Channel DG FETs}

The $\mathrm{Na}$ ionophore $\mathrm{X}$ of the sodium-selective membrane captures the sodium ions from $\mathrm{NaCl}$ molecules and modifies the $\psi_{0}$ of the $\mathrm{SnO}_{2}$-sensing dielectric film, upon the exposure of the sodium-selective membrane of the EG to a solution of $\mathrm{NaCl}[20,21]$. The $\Delta \psi_{0}$ of the $\mathrm{SnO}_{2}$-sensing dielectric film is applied to the Al top gate of the FET transducer, which is electrically connected in series through the ITO conductive film of the EG. Therefore, the sensitivity of the SG mode depends only on $\psi_{0}$, whereas the sensitivity of the DG mode is given by the value of $\psi_{0}$ amplified by the capacitive coupling ratio, as shown in Equation (1).

Figure 8 shows the transfer characteristic curves of a sodium ion sensor based on a SiNW channel DG FET with a sodium-selective membrane EG. The sensing characteristics for the $\mathrm{NaCl}, \mathrm{CaCl}_{2}, \mathrm{KCl}$, and $\mathrm{pH}$ buffer solutions during the $\mathrm{SG}$ mode operations are shown in Figure $8 \mathrm{a}-\mathrm{d}$, respectively. Figure $8 \mathrm{e}-\mathrm{h}$ present the sensing characteristics during the DG mode operations for the same solutions. It is noteworthy that the distinct shift in the transfer characteristic curve of the sensing characteristics was only observed for the $\mathrm{NaCl}$ solution. It means that the sodium-selective membrane only reacts with the sodium ions of $\mathrm{NaCl}$. A reduction in the concentration shifts the transfer curves in the positive direction.

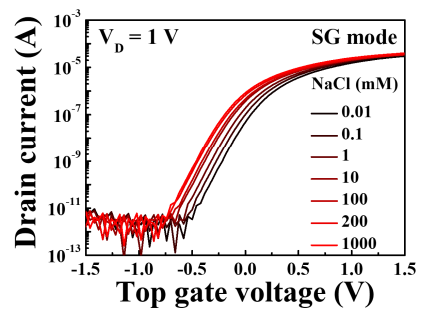

(a)

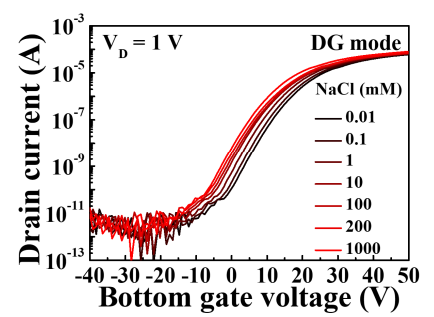

(e)

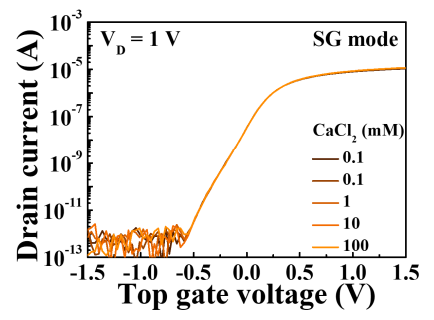

(b)

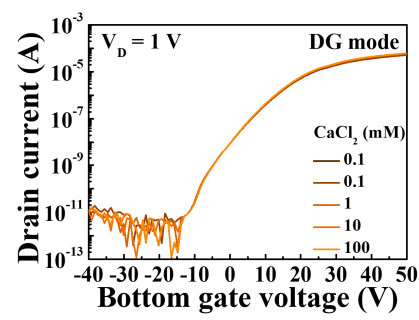

(f)

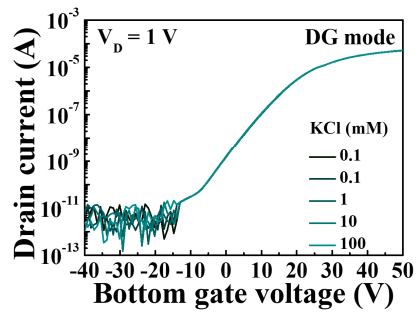

(c)

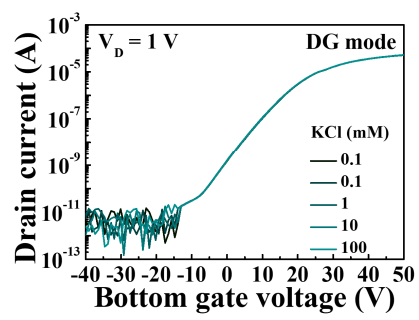

(g)

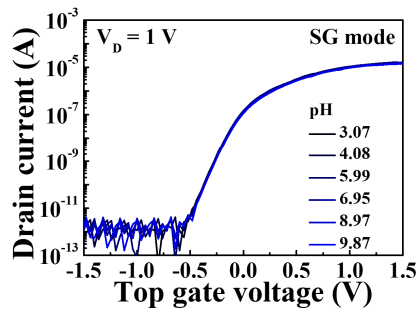

(d)

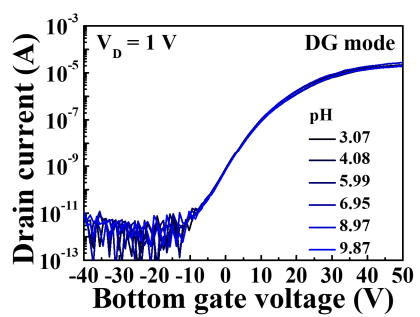

(h)

Figure 8. Transfer characteristic curves of the sodium ion sensor based on a SiNW channel DG FET with a sodium-selective membrane EG in various buffer solutions: SG mode operations for the (a) $\mathrm{NaCl}$, (b) $\mathrm{CaCl}_{2}$, (c) $\mathrm{KCl}$, and (d) $\mathrm{pH}$ buffer

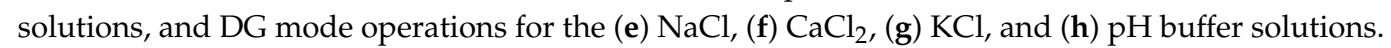


Figure 9a,b, which were obtained from the transfer characteristic curves of the SG and DG mode operations, respectively, display the variation in the electrolyte concentration with the $\Delta \mathrm{V}_{\text {ref }}$ of the sodium ion sensor. The slope of linear fitting of the $\Delta \mathrm{V}_{\text {ref }}$ plot represents the sensitivity. As can be seen in Figure 8a,e, the transfer characteristic curves shifted at regular intervals only in the $10^{-4} \sim 10^{0} \mathrm{M}$ section. It showed poor linearity at $10^{-5} \mathrm{M}$, which means that the limit of detection is about $10^{-4} \mathrm{M}$. Therefore, we extracted the sensitivity in the $10^{-4} \sim 10^{0} \mathrm{M}$ section. The SiNW channel FET-based sodium ion sensor implemented in this study has a sensitivity of $57.33 \mathrm{mV} / \mathrm{dec}$ with respect to $\mathrm{NaCl}$ in the SG mode, which is similar to that of a conventional FET-based sodium sensor. However, an extremely high sensitivity of $1464.66 \mathrm{mV} /$ dec was achieved in the DG mode, which is 26.6 times the maximum sensitivity of a conventional FET-based sodium sensor $(\sim 55 \mathrm{mV} / \mathrm{dec})$. As a result of evaluating the Na-sensing ability for $\mathrm{mV}$ by taking the reciprocal of the sensitivity, the SG mode and DG mode are $1.74 \times 10^{-2} \mathrm{dec} / \mathrm{mV}$ and $6.85 \times 10^{-4} \mathrm{dec} / \mathrm{mV}$, respectively. The goal of sensitivity amplification is to reduce resolution, which means the smallest change in concentration that the sensor can detect. Therefore, in terms of the resolution of the sodium ion sensor, it is evident that DG mode operation is much more advantageous than SG mode operation due to the capacitive coupling. In addition, the linearity of the $\Delta \mathrm{V}_{\text {ref }}$ plots including $100 \mathrm{mM}$ and $200 \mathrm{mM}$, which is related to physiological concentration, was $99.7 \%$ and $99.8 \%$ in SG mode and DG mode, respectively. Linearity means how linearly the $\Delta \mathrm{V}_{\text {ref }}$ plot is distributed and how $\Delta \mathrm{V}_{\text {ref }}$ is constantly increasing. The slope of the $\Delta \mathrm{V}_{\text {ref }}$ plot fitting line represents the sensitivity and means the $\mathrm{mV}$ shift of the transfer characteristic curve per concentration. Therefore, $\Delta \mathrm{V}_{\text {ref }}$ for different concentrations can be predicted through sensitivity, and the higher the linearity of the $\Delta \mathrm{V}_{\text {ref }}$ plots, the better the reliability. The sodium ion sensor proposed in this paper showed excellent sensing performance with high sensitivity and excellent linearity. Moreover, the responses of the SiNW channel FET-based sodium ion sensor to the $\mathrm{CaCl}_{2}, \mathrm{KCl}$, and $\mathrm{pH}$ buffer solutions were relatively insignificant. The sensitivities in the SG and DG modes did not exceed the Nernstian limit. Therefore, the proposed sodium ion sensor is highly selective to sodium owing to its extremely high sensitivity to $\mathrm{NaCl}$ buffer solutions, especially in the DG mode. However, its sensitivity to the $\mathrm{CaCl}_{2}, \mathrm{KCl}$, and $\mathrm{pH}$ buffer solutions was considerably low, which implies that the sodium-selective membrane has excellent selectivity for other ions.

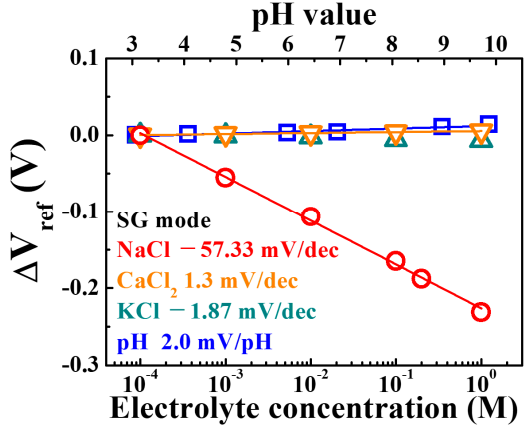

(a)

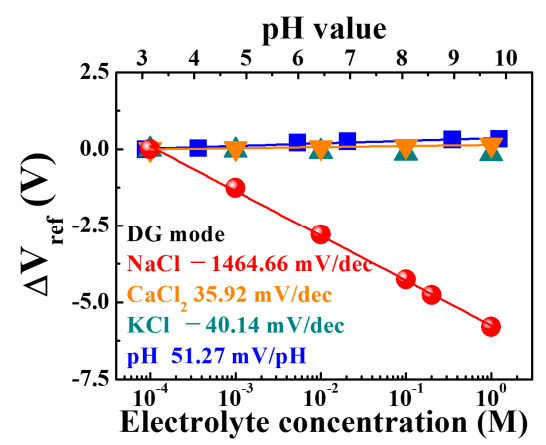

(b)

Figure 9. Sensitivity of the sodium ion sensor based on a SiNW channel DG FET with sodium-selective membrane EG in various buffer solutions during the (a) SG mode and (b) DG mode operations.

In addition, based on comprehensive theoretical analyses of DG structures, previous studies have reported that DG FET-based sensors outperform SG FET-based sensors in terms of the signal-to-noise ratio (SNR) and resolution [22]. The SNR of a sensor is expressed as the ratio of signal power to the noise power. Although instrument noise is similar for both SG FET and DG FET, DG FET is more advantageous in terms of SNR because DG FET has higher sensitivity than SG FET. Therefore, SiNW channel DG FETs not 
only achieve high sensitivity through capacitive coupling, but also serve as a stable sensor platform against noise.

\subsection{Reliability and Stability Evaluation of Sodium Ion Sensors}

As the non-ideal operations of chemical sensors limit their accuracies, it is important to ensure their reliability and stability in repetitive and long-term operations, in order to implement high-performance sensors. In this study, the hysteresis and drift effects were measured to evaluate the reliability and stability of the sodium ion sensor based on the SiNW channel DG FET with a sodium-selective membrane EG. The hysteresis effect was used to evaluate reliability by measuring the variation in the sensing properties of the sensor under exposure to varying solution concentrations. Similarly, the drift effect was used to evaluate stability by measuring the variation in the sensing behavior of the sensor under prolonged exposure [23-26].

Figure 10a,b show the hysteresis effects of a sodium ion sensor based on a SiNW channel DG FET with a sodium-selective membrane EG operating in the SG and DG modes, respectively. Following a concentration loop of $10^{-4} \rightarrow 10^{-3} \rightarrow 10^{-2} \rightarrow 10^{-1} \rightarrow$ $10^{0} \rightarrow 10^{-1} \rightarrow 10^{-2} \rightarrow 10^{-3} \rightarrow 10^{-4} \mathrm{M}$, the hysteresis voltages were $2.5 \mathrm{mV}$ and $12.4 \mathrm{mV}$ in the SG and DG modes, respectively. These values were significantly lower than its sensitivity to sodium. The hysteresis voltages of the SG and DG modes were $4.3 \%$ and $0.8 \%$ of the sensitivity voltage, respectively. This indicates that the SiNW channel DG FET-based sodium ion sensor with a sodium-selective membrane EG is more reliable in the DG mode than in the SG mode owing to its high sensitivity.

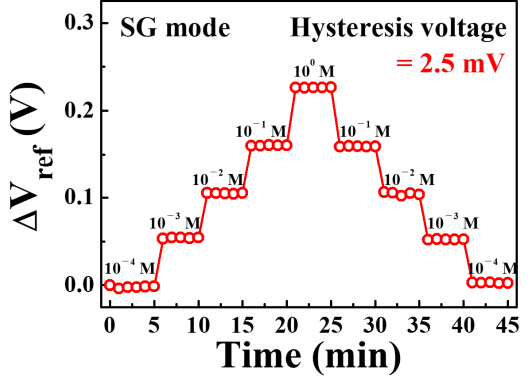

(a)

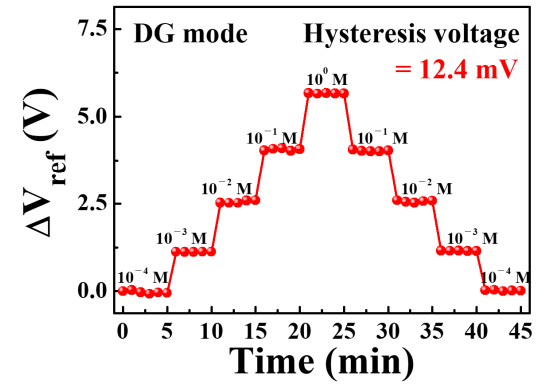

(b)

Figure 10. Hysteresis effects of the sodium ion sensor based on SiNW channel DG FETs with sodium-selective membrane EG during the (a) SG mode and (b) DG mode operations.

Figure 11a,b show the drift effects of the sodium ion sensor based on a SiNW channel DG FET with a sodium-selective membrane EG operating in the SG and DG modes, respectively. The drift rates of the sodium ion sensor after exposure to a sodium electrolyte concentration of $10^{-4} \mathrm{M}$ for $10 \mathrm{~h}$ were $2.7 \mathrm{mV} / \mathrm{h}$ and $17.2 \mathrm{mV} / \mathrm{h}$ in the SG and DG modes, respectively. The drift rates in the SG and DG modes were low in comparison to the sensitivity, similar to the observations for the hysteresis voltage. The drift rates in the SG and DG modes were $4.7 \%$ and $1.2 \%$ of the sensitivity, respectively. This indicates that the SiNW channel DG FET-based sodium ion sensor with a sodium-selective membrane EG is more stable and reliable in the DG mode than in the SG mode owing to its relatively high sensitivity in the former. 


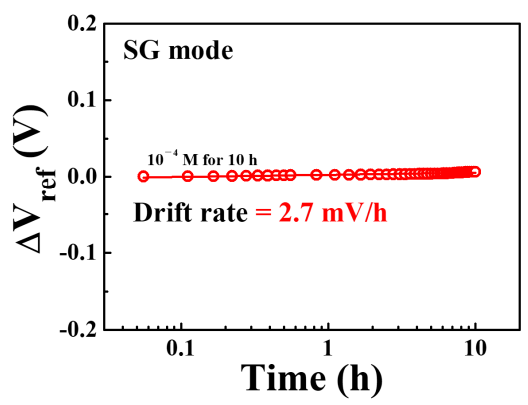

(a)

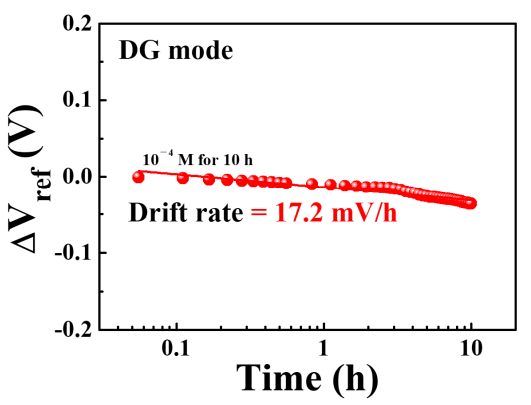

(b)

Figure 11. Drift effects of the sodium ion sensor based on SiNW channel DG FETs with a sodium-selective membrane EG after exposure to a sodium electrolyte concentration of $10^{-4} \mathrm{M}$ for $10 \mathrm{~h}$ during the (a) SG mode and (b) DG mode operations.

\section{Conclusions}

In this paper, a highly sensitive sodium ion sensor with excellent sensitivity was proposed; the sensor comprised a SiNW channel DG FET and a sodium-selective membrane EG as the transducer and sensing units, respectively. A DG structure FET consisting of a SiNW channel was fabricated as a transducer device by using a simple and inexpensive PVP NF pattern template transfer method, in order to maximize capacitive coupling and amplify sensitivity through capacitive coupling. The fabricated SiNW channel DG FET exhibited excellent electrical performance and signal amplification. In addition, a sodiumselective membrane EG was fabricated with a sensing device that selectively detected only sodium ions; the $\mathrm{Na}$ ionophore $\mathrm{X}$ in the sodium-selective membrane only captured the sodium ions from solutions and altered the surface potential of the $\mathrm{SnO}_{2}$ sensing dielectric films. A sodium ion sensor was constructed by electrically connecting the sodium-selective membrane EG to the SiNW channel DG FET in series. An extremely high sensitivity of $1464.66 \mathrm{mV} / \mathrm{dec}$ was obtained for $\mathrm{NaCl}$ solutions in the DG mode. However, the sensitivities to the $\mathrm{CaCl}_{2}, \mathrm{KCl}$, and $\mathrm{pH}$ buffer solutions were significantly lower than that for the $\mathrm{NaCl}$ buffer solution. This proved the excellent selectivity of the sensor. Furthermore, the hysteresis voltage and the drift rate under non-ideal conditions were $12.4 \mathrm{mV}$ and $17.2 \mathrm{mV} / \mathrm{h}$, respectively, thereby confirming the excellent reliability and stability of the sensor, in addition to its enhanced sodium ion sensitivity. These results suggest that the proposed sodium ion sensor is not only highly sensitive and selective toward sodium ions, but also reliable for repetitive and long-term operations. Thus, the SiNW channel DG FET-based sodium ion sensor platform with the sodium-selective membrane EG can be used for the precise and selective detection of sodium ions in the bioanalysis of sweat or blood.

Author Contributions: S.-K.C.: conceptualization, formal analysis, methodology, investigation, data curation, visualization, software, resources, writing-original draft. W.-J.C.: conceptualization, methodology, investigation, resources, formal analysis, funding acquisition, supervision, validation, writing-review and editing. Both authors have read and agreed to the published version of the manuscript.

Funding: The study was supported by the Research Grant of Kwangwoon University in 2021 and the National Research Foundation of Korea (NRF) grant funded by the Korea government (MSIT) (No. 2020R1A2C1007586). The work was also funded and conducted under [the Competency Development Program for Industry Specialists] of the Korean Ministry of Trade, Industry and Energy (MOTIE), operated by Korea Institute for Advancement of Technology (KIAT) (No. P0002397, HRD program for Industrial Convergence of Wearable Smart Devices).

Conflicts of Interest: The authors declare no conflict of interest. 


\section{References}

1. Hsu, H.Y.; Wu, C.Y.; Lee, H.C.; Lin, J.L.; Chin, Y.L.; Sun, T.P. Sodium and potassium sensors based on separated extended gate field effect transistor. Biomed. Eng. Appl. Basis Commun. 2009, 21, 441-444. [CrossRef]

2. Melzer, K.; Münzer, A.M.; Jaworska, E.; Maksymiuk, K.; Michalska, A.; Scarpa, G. Selective ion-sensing with membranefunctionalized electrolyte-gated carbon nanotube field-effect transistors. Analyst 2014, 139, 4947-4954. [CrossRef] [PubMed]

3. Kim, S.J.; Jung, J.; Lee, K.W.; Yoon, D.H.; Jung, T.S.; Dugasani, S.R.; Park, S.H.; Kim, H.J. Low-cost label-free electrical detection of artificial DNA nanostructures using solution-processed oxide thin-film transistors. ACS Appl. Mater. Interfaces 2013, 5, 10715-10720. [CrossRef]

4. Matsumoto, A.; Miyahara, Y. Current and emerging challenges of field effect transistor based bio-sensing. Nanoscale 2013, 5, 10702-10718. [CrossRef]

5. Sarkar, D.; Liu, W.; Xie, X.; Anselmo, A.C.; Mitragotri, S.; Banerjee, K. MoS 2 field-effect transistor for next-generation label-free biosensors. ACS Nano 2014, 8, 3992-4003. [CrossRef]

6. Ma, S.; Lee, Y.K.; Zhang, A.; Li, X. Label-free detection of Cordyceps sinensis using dual-gate nanoribbon-based ion-sensitive field-effect transistor biosensor. Sens. Actuators B Chem. 2018, 264, 344-352. [CrossRef]

7. Knopfmacher, O.; Tarasov, A.; Fu, W.; Wipf, M.; Niesen, B.; Calame, M.; Schonenberger, C. Nernst limit in dual-gated Si-nanowire FET sensors. Nano Lett. 2010, 10, 2268-2274. [CrossRef]

8. Spijkman, M.; Smits, E.C.P.; Cillessen, J.F.M.; Biscarini, F.; Blom, P.W.M.; De Leeuw, D.M. Beyond the Nernst-limit with dual-gate ZnO ion-sensitive field-effect transistors. Appl. Phys. Lett. 2011, 98, 043502. [CrossRef]

9. Spijkman, M.J.; Myny, K.; Smits, E.C.; Heremans, P.; Blom, P.W.; De Leeuw, D.M. Dual-gate thin-film transistors, integrated circuits and sensors. Adv. Mater. 2011, 23, 3231-3242. [CrossRef]

10. Lim, H.K.; Fossum, J.G. Threshold voltage of thin-film silicon-on-insulator (SOI) MOSFET's. IEEE Trans. Electron Devices 1983, 30, 1244-1251.

11. Jang, H.J.; Cho, W.J. Performance enhancement of capacitive-coupling dual-gate ion-sensitive field-effect transistor in ultra-thinbody. Sci. Rep. 2014, 4, 5284. [CrossRef]

12. Lee, I.K.; Lee, K.H.; Lee, S.; Cho, W.J. Microwave annealing effect for highly reliable biosensor: Dual-gate ion-sensitive field-effect transistor using amorphous InGaZnO thin-film transistor. ACS Appl. Mater. Interfaces 2014, 6, 22680-22686. [CrossRef]

13. Lee, I.K.; Jeun, M.; Jang, H.J.; Cho, W.J.; Lee, K.H. A self-amplified transistor immunosensor under dual gate operation: Highly sensitive detection of hepatitis B surface antigen. Nanoscale 2015, 7, 16789-16797. [CrossRef]

14. Jang, H.J.; Gu, J.G.; Cho, W.J. Sensitivity enhancement of amorphous InGaZnO thin film transistor based extended gate field-effect transistors with dual-gate operation. Sens. Actuators B Chem. 2013, 181, 880-884. [CrossRef]

15. Cho, S.K.; Cho, W.J. Ultra-high sensitivity pH-sensors using silicon nanowire channel dual-gate field-effect transistors fabricated by electrospun polyvinylpyrrolidone nanofibers pattern template transfer. Sens. Actuators B Chem. 2021, 326, 128835. [CrossRef]

16. Schmiers, H.; Friebel, J.; Streubel, P.; Hesse, R.; Köpsel, R. Change of chemical bonding of nitrogen of polymeric N-heterocyclic compounds during pyrolysis. Carbon 1999, 37, 1965-1978. [CrossRef]

17. Chen, S.; Bomer, J.G.; Carlen, E.T.; van den Berg, A. $\mathrm{Al}_{2} \mathrm{O}_{3}$ / silicon nanoISFET with near ideal Nernstian response. Nano Lett. 2011, 11, 2334-2341. [CrossRef] [PubMed]

18. Ahn, M.J.; Lim, C.M.; Cho, W.J. Highly sensitive ion-sensitive field-effect transistor sensor using fully transparent amorphous In-Ga-Zn-O thin-film transistors. Semicond. Sci. Technol. 2017, 32, 035003. [CrossRef]

19. Masahara, M.; Liu, Y.; Sakamoto, K.; Endo, K.; Matsukawa, T.; Ishii, K.; Sekigawa, T.; Yamauchi, H.; Tanoue, H.; Kanemaru, S.; et al. Demonstration, analysis, and device design considerations for independent DG MOSFETs. IEEE Trans. Electron Devices 2005, 52, 2046-2053. [CrossRef]

20. Garcia-Cordero, E.; Bellando, F.; Zhang, J.; Wildhaber, F.; Longo, J.; Guerin, H.; Ionescu, A.M. Three-dimensional integrated ultra-low-volume passive microfluidics with ion-sensitive field-effect transistors for multiparameter wearable sweat analyzers. ACS Nano 2018, 12, 12646-12656. [CrossRef]

21. Zhang, J.; Rupakula, M.; Bellando, F.; Garcia Cordero, E.; Longo, J.; Wildhaber, F.; Herment, G.; Guerin, H.; Ionescu, A.M. Sweat biomarker sensor incorporating picowatt, three-dimensional extended metal gate ion sensitive field effect transistors. ACS Sens. 2019, 4, 2039-2047. [CrossRef]

22. Chang, S.P.; Yang, T.H. Sensing performance of EGFET pH sensors with $\mathrm{CuO}$ nanowires fabricated on glass substrate. Int. J. Electrochem. Sci. 2012, 7, 5020-5027.

23. Fung, C.D.; Cheung, P.W.; Ko, W.H. A generalized theory of an electrolyte-insulator-semiconductor field-effect transistor. IEEE Trans. Electron Devices 1986, 33, 8-18. [CrossRef]

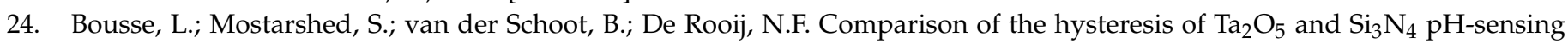
insulators. Sens. Actuators B Chem. 1994, 17, 157-164. [CrossRef]

25. Chiang, J.L.; Jan, S.S.; Chou, J.C.; Chen, Y.C. Study on the temperature effect, hysteresis and drift of pH-ISFET devices based on amorphous tungsten oxide. Sens. Actuators B Chem. 2001, 76, 624-628. [CrossRef]

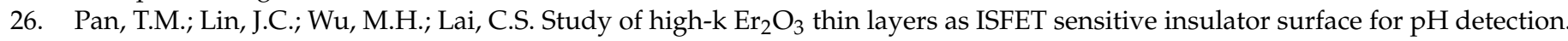
Sens. Actuators B Chem. 2009, 138, 619-624. [CrossRef] 